\title{
AN EXTENSION OF THE NOETHER-DEURING THEOREM
}

\author{
KLAUS W. ROGGENKAMP
}

\begin{abstract}
Let $R$ be a commutative semilocal noetherian ring, $\Lambda$ a left noetherian $R$-algebra and $M, N$ finitely generated left $\Lambda$-modules such that $\operatorname{End}_{\Lambda}(M)$ is of finite type over $R$. By $\hat{R}$ we denote the ( $\operatorname{rad} R)$-adic completion of $R$.

THEOREM. $M$ is $\Lambda$-isomorphic to a direct summand of $N$ iff $\hat{R} \otimes_{R} M$ is $\hat{R} \otimes_{R} \Lambda$-isomorphic to a direct summand of $\hat{R} \otimes_{R} N$.

This result is used to prove a generalization of the NOETHER-DEURING THEOREM. Let $S$ be a commutative $R$-algebra which is a faithful projective $R$-module of finite type; then $M$ is $\Lambda$-isomorphic to direct summand of $N$ iff $S \otimes_{R} M$ is $S \otimes_{R} \Lambda$-isomorphic to a direct summand of $S \otimes_{R} N$.
\end{abstract}

Let $R$ be a semilocal commutative noetherian ring with Jacobson radical $J(R)$ and denote by $\hat{R}$ the $J(R)$-adic completion of $R$; let $S$ be a commutative $R$-algebra such that $\hat{S}=\hat{R} \otimes_{R} S$ is a faithful projective $\hat{R}$-module of finite type. As a generalization of the Noether-Deuring theorem for integral representations we shall prove

Theorem I. Let $\Lambda$ be a left noetherian R-algebra, and $M, N$ finitely generated left $\Lambda$-modules such that $\operatorname{End}_{\Lambda}(M)$ is of finite type over $R$. Then $M$ is $\Lambda$-isomorphic to a direct summand of $N$ if and only if $S \otimes_{R} M$ is $S \otimes_{R} \Lambda$-isomorphic to a direct summand of $S \otimes_{R} N$.

It has been pointed out to me by the referee that this is part of a result of A. Grothendieck [8, Proposition 2.5.8.(a)]. A similar statement has also been proven by Białynicki-Birula [4] using noncommutative Amitsur cohomology and the "théorie de déscente". Our theorem here is the result of an attempt to give a simplified proof of one of the theorems in Białynicki-Birula's paper.

We shall keep the notation introduced above throughout the paper, and to simplify the notation, we shall write $X \mid Y$ to indicate that $X$ is isomorphic to a direct summand of $Y$. The key role in our proof of

Received by the editors June 8, 1970 and, in revised form, November 23, 1970.

AMS 1969 subject classifications. Primary 1640, 1395; Secondary 2080.

Key words and phrases. Algebra, semilocal noetherian ring, finitely presented module, isomorphism, completion, Noether-Deuring theorem.

(c) American Mathematical Society 1972 
Theorem I is played by

THEOREM II. Let $\Lambda$ be a left noetherian R-algebra, and $M, N$ finitely generated left $\Lambda$-modules such that $\operatorname{End}_{\Lambda}(M)$ is of finite type over $R$. Then

$$
\begin{array}{r}
M \mid N \text { as } \Lambda \text {-modules if and only if } \\
\hat{R} \otimes_{R} M \mid \hat{R} \otimes_{R} N \text { as } \hat{R} \otimes_{R} \Lambda \text {-modules. }
\end{array}
$$

We remark that in both theorems the condition that $\operatorname{End}_{\Lambda}(M)$ is of finite type over $R$ is surely satisfied if $M$ is of finite type over $R$; in fact, $R^{(s)} \rightarrow M \rightarrow 0$ exact, implies $0 \rightarrow \operatorname{End}_{R}(M) \rightarrow M^{(s)}$ exact, and so $\operatorname{End}_{R}(M)$ is of finite type over $R, R$ being noetherian. But $\operatorname{End}_{\Lambda}(M) \leftrightarrow \operatorname{End}_{R}(M)$ and so $\operatorname{End}_{\Lambda}(M)$ is of finite type over $R$.

Proof of Theorem II. It suffices to prove one direction. So let us assume $\hat{R} \otimes_{R} M \mid \hat{R} \otimes_{R} N$ as $\hat{R} \otimes_{R} \Lambda$-modules. This is equivalent to the existence of a split monomorphism

$$
0 \longrightarrow \hat{R} \otimes_{R} M \stackrel{\hat{\sigma}}{\rightarrow} \hat{R} \otimes_{R} N .
$$

Since $\hat{R}$ is a faithfully flat $R$-module (cf. Bourbaki [6, Chapitre III, $\S 3$, $\left.\mathrm{N}^{\circ} 5\right]$ ) and since $M$ is a finitely generated left module over the left noetherian ring $\Lambda$, we have natural isomorphisms (cf. Auslander-Goldman [1, Lemma 2.4]) for any left $\Lambda$-module $X$.

(1) $\hat{R} \otimes_{R} \operatorname{Ext}_{\Lambda}^{i}(M, X) \cong \operatorname{Ext}_{\hat{R} \otimes_{R} \Lambda}^{i}\left(\hat{R} \otimes_{R} M, \hat{R} \otimes_{R} X\right)$ for $1=0,1, \cdots$.

Using this isomorphism for $i=0$ and identifying both structures, we may write

$$
\hat{\sigma}=\sum_{i=1}^{n} \hat{r}_{i} \otimes \sigma_{i}, \quad \hat{r}_{i} \in \hat{R}, \sigma_{i} \in \operatorname{Hom}_{\Lambda}(M, N), 1 \leqq i \leqq n .
$$

However, $R / J(R) \cong \hat{R} / \hat{R} \otimes_{R} J(R)$, and so we can find elements $r_{i} \in R$, $1 \leqq i \leqq n$, such that $1 \wedge \otimes r_{i}-\hat{r}_{i} \in \hat{R} \otimes_{R} J(R)=J(\hat{R})$. To prove Theorem II, we have to establish the existence of a split monomorphism $0 \rightarrow M_{\rightarrow}^{\sigma} N$. We claim that $\sigma=\sum_{i=1}^{n} r_{i} \varphi_{i} \in \operatorname{Hom}_{\Lambda}(M, N)$ has the desired properties. Since $\hat{R}$ is a faithfully flat $R$-module, it suffices to show that $1_{\hat{R}} \otimes_{R} \sigma$ is a split monomorphism. In fact, assuming that $1_{R} \otimes_{R} \sigma$ is a split monomorphism, $\sigma$ must be monic, and it remains to show that the sequence

$$
E: 0 \longrightarrow M \stackrel{\sigma}{\longrightarrow} N \longrightarrow N / \operatorname{Im} \sigma \rightarrow 0
$$

is split exact. We consider the $R$-submodule $X$ of $\operatorname{Ext}_{\Lambda}^{1}(N / \operatorname{Im} \sigma, M)$ generated by the class of $E$. Because of the isomorphism (1) for $i=1$ we have $\hat{R} \otimes_{R} X=0$ and so $X$ must be zero; i.e., $E$ is split exact. It remains to show that $1_{\hat{R}} \otimes \sigma$ is a split monomorphism. Since $\hat{\sigma}$ was a split monomorphism to start with, there exists $\hat{\tau} \in \operatorname{Hom}_{\hat{R} \otimes_{R} \Lambda}\left(\hat{R} \otimes_{R} N, \hat{R} \otimes_{R} M\right)$ such 
that $\hat{\sigma} \hat{\tau}=1_{\hat{R} \otimes_{R} M}$. But then

$$
\begin{aligned}
\left(1_{\hat{R}} \otimes \sigma\right) \hat{\tau}-1_{\hat{R} \otimes_{R} M} & =\left(1_{\hat{R}} \otimes \sigma-\hat{\sigma}\right) \hat{\tau} \\
& =\left[\sum_{i=1}^{n}\left(r_{i}-\hat{r}_{i}\right) \otimes \sigma_{i}\right] \hat{\tau} \in J(\hat{R}) \operatorname{End}_{\hat{R} \otimes_{R} \Lambda}\left(\hat{R} \otimes_{R} M\right) .
\end{aligned}
$$

But $\operatorname{End}_{\hat{R} \otimes_{R} \Lambda}\left(\hat{R} \otimes_{R} M\right)$ is of finite type over $\hat{R}$ and so $J(\hat{R}) \operatorname{End}_{\hat{R} \otimes_{R} \Lambda}(\hat{R} \otimes M)$ is contained in the Jacobson radical of $\operatorname{End}_{\hat{R} \otimes_{R} \Lambda}\left(\hat{R} \otimes_{R} M\right)$ (cf. Bourbaki [5, Chapitre VIII, $\S 6, \mathrm{~N}^{\circ} 3$, Théorème 2]) and so $\left(1_{\hat{R}} \otimes \sigma\right) \hat{\tau}$ is a unit in End $_{\hat{R} \otimes \Lambda}\left(\hat{R} \otimes_{R} M\right)$; i.e., $1_{\hat{R}} \otimes \sigma$ is a split monomorphism and so $M \mid N$. Q.E.D.

COROLlaRY 1. Let $\Lambda$ be an R-algebra and $M$ a finitely presented left $\Lambda$-module such that $\operatorname{End}_{\Lambda}(M)$ is of finite type over $R$ and $N$ a left $\Lambda$-module. Then $M \cong{ }_{\Lambda} N$ if and only if $\hat{R} \otimes_{R} M \cong \hat{R} \otimes_{R} N$.

The proof is similar to the one of Theorem II; however, we do not need the assumption that $\Lambda$ is left noetherian, since (1) is valid for $i=0$ also for a finitely presented $\Lambda$-module $M$.

REMARK. Under the assumptions of Corollary 1, the Krull-Schmidt theorem is valid for the indecomposable direct summands of $\hat{R} \otimes_{R} M$. For this it suffices to know, that for each indecomposable direct summand $\hat{X}$ of $\hat{R} \otimes_{R} M$, the ring $\operatorname{End}_{\hat{R} \otimes_{R} \Lambda}(\hat{X})$ is complete with respect to the topology induced by $J(\hat{R})$ End $_{\hat{R} \otimes_{R} \Lambda}(\hat{X})$ (cf. Bass [3, Chapter III, Proposition 2.10]); but this is clear since $\operatorname{End}_{\hat{R} \otimes_{R} \Lambda}(\hat{X})$ is of finite type over $\hat{R}$ (cf. Bourbaki [6, Chapitre III, $\S 3, \mathrm{~N}^{\circ} 4$, Théorème 3]).

COROLlaRY 2. Under the assumptions of Corollary 1, let $X$ be a finitely presented left $\Lambda$-module such that $\operatorname{End}_{\Lambda}(M \oplus X)$ is of finite type over $R$. Then $M \oplus X \cong N \oplus X$ implies $M \cong N$.

Proof. This is an immediate consequence of Corollary 1 and the Remark.

COROLlaRY 3. Under the assumptions of Corollary $1, M^{(n)} \cong N^{(n)}$ implies $M \cong N$.

Proof. This follows from Corollary 1 and the Remark.

COROLlaRy 4. Let $\mu_{\Lambda}(X)$ denote the minimal number of $\Lambda$-generators of the left $\Lambda$-module $X$, where $\Lambda$ is an $R$-algebra. Assume that $M$ is a finitely presented left $\Lambda$-module which is of finite type over $R$. Then $\mu_{\Lambda}(M) \leqq n$ if and only if $\mu_{\hat{R} \otimes_{R} \Lambda}\left(\hat{R} \otimes_{R} M\right) \leqq n$.

Proof. Since the tensor product is right exact, it suffices to prove one direction. Let $\mu_{\hat{R} \otimes_{R} \Lambda}\left(\hat{R} \otimes_{R} M\right) \leqq n$. Then we have an epimorphism

$$
\left(\hat{R} \otimes_{R} \Lambda\right)^{(n)} \stackrel{\hat{\sigma}}{\longrightarrow} \hat{R} \otimes_{R} M \longrightarrow 0 .
$$


As in the proof of Theorem II, we construct $\sigma \in \operatorname{Hom}_{\Lambda}\left(\Lambda^{(n)}, M\right)$ such that

$$
\left(1_{\hat{R}} \otimes \sigma\right)-\hat{\sigma} \in J(\hat{R}) \operatorname{Hom}_{\hat{R} \otimes_{R} \Lambda}\left(\hat{R} \otimes_{R} \Lambda^{(n)}, \hat{R} \otimes_{R} M\right) .
$$

But then $\operatorname{Im}\left(1_{\hat{R}} \otimes \sigma\right)+J(\hat{R})\left(\hat{R} \otimes_{R} M\right)=\hat{R} \otimes_{R} M$ and Nakayama's Lemma shows that $1_{\hat{R}} \otimes \sigma$ must be an epimorphism. However, $\hat{R} \otimes_{R}-$ is faithfully flat, and so $\varphi$ is an epimorphism.

Finally we turn to the proof of Theorem I.

It suffices to prove one direction. Let $S \otimes_{R} M \mid S \otimes_{R} N$. Then $\hat{S} \otimes_{R} M \mid \hat{S} \otimes_{R} N$ as $\hat{S} \otimes_{R} \Lambda$-modules. However, $J(R)=\bigcap_{i=1}^{s} m_{i}$, where $\left\{m_{i}\right\}_{1 \leqq i \leqq s}$ are the maximal ideals of $R$. Then $\hat{R}=\prod_{i=1}^{s} \hat{R}_{i}$ is the product of complete local rings. Since we have assumed $\hat{S}$ to be a faithful projective $\hat{R}$-module of finite type, we have

$$
\hat{S} \cong \hat{R} \bigoplus_{i=1}^{s} \hat{R}_{i}^{(n)_{i}}, \quad n_{i}>0, \quad 1 \leqq i \leqq s .
$$

Thus $\hat{S} \otimes_{R} M \mid \hat{S} \otimes_{R} N$ as $\hat{S} \otimes_{R} \Lambda$-modules implies

$$
\left(\hat{R}_{i} \otimes_{R} M\right)^{\left(n_{i}\right)} \mid\left(\hat{R}_{i} \otimes_{R} N\right)^{\left(n_{i}\right)}, \quad 1 \leqq i \leqq s
$$

as $\hat{R}_{i} \otimes_{R} \Lambda$-modules. Now the Krull-Schmidt theorem shows

$$
\hat{R}_{i} \otimes_{R} M \mid \hat{R}_{i} \otimes_{R} N, \quad 1 \leqq i \leqq s,
$$

and so $\hat{R} \otimes_{R} M \mid \hat{R} \otimes_{R} N$. An application of Theorem II gives the desired result: $M \mid N$. Q.E.D.

\section{REFERENCES}

1. M. Auslander and O. Goldman, Maximal orders, Trans. Amer. Math. Soc. 97 (1960), 1-24. MR 22 \#8034.

2. H. Bass, $K$-theory and stable algebra, Inst. Hautes Études Sci. Publ. Math. No. 22 (1964), 5-60. MR 30 \#4805.

3. - Algebraic K-theory, Benjamin, New York, 1968. MR 40 \#2736.

4. A. Białynicki-Birula, On the equivalence of integral representations of groups, Proc. Amer. Math. Soc. 26 (1970), 371-377.

5. N. Bourbaki, Algèbre, Actualités Sci. Indust., no. 1272, Hermann, Paris, 1959. MR 21 \#6384.

6. - - Algèbre commutative. Chap. 3, Actualités Sci. Indust., no. 1293, Hermann, Paris, 1961. MR 30 \#2027.

7. A. Dress, On the decomposition of modules, Bull. Amer. Math. Soc. 75 (1969), 984-986. MR 39 \#5544.

8. A. Grothendieck, Eléments de géométrie algébrique. IV. Étude locale des schémas et des morphismes de schémas. II, Inst. Hautes Études Sci. Publ. Math. No. 24 (1965), 231 pp. MR 33 \#7330.

9. R. G. Swan, The number of generators of a module, Math. Z. 102 (1967), 318-322. MR 36 \#1434.

Mathematische Fakultät, Universität Bielefeld, 48 Bielefeld, Kurt-SchuMACHERSTRASSE 6, WeST GeRMANY 